\title{
A Linear Programming Relaxation DEA Model for Selecting a Single Efficient Unit with Variable RTS Technology
}

\author{
Reza Akhlaghi ${ }^{1}$, Mohsen Rostamy-Malkhalifeh ${ }^{2, *}$, Alireza Amirteimoori ${ }^{1}$ and \\ Sohrab Kordrostami ${ }^{3}$ \\ ${ }^{1}$ Department of Applied Mathematics, Rasht Branch, Islamic Azad University, Rasht, Iran \\ E-mail: 〈mathe8877@gmail.com〉,〈aamirteimoori@gmail.com〉 \\ ${ }^{2}$ Department of Mathematics, Tehran Science and Research Branch, Islamic Azad University, \\ Tehran, Iran \\ E-mail: 〈mohsen_rostamy@yahoo.com〉 \\ ${ }^{3}$ Department of Applied Mathematics, Lahijan Branch, Islamic Azad University, Lahijan, Iran \\ E-mail: 〈sohrabkordrostami@gmail.com〉
}

\begin{abstract}
The selection-based problem is a type of decision-making issue which involves opting for a single option among a set of available alternatives. In order to address the selection-based problem in data envelopment analysis (DEA), various integrated mixed binary linear programming (MBLP) models have been developed. Recently, an MBLP model has been proposed to select a unit in DEA with variable returns-to-scale technology. This paper suggests utilizing the linear programming relaxation model rather than the MBLP model. The MBLP model is proved here to be equivalent to its linear programming relaxation problem. To the best of the authors' knowledge, this is the first linear programming model suggested for selecting a single efficient unit in DEA under the VRS (Variable Returns to Scale) assumption. Two theorems and a numerical example are provided to validate the proposed LP model from both theoretical and practical perspectives.
\end{abstract}

Keywords: data envelopment analysis (DEA), selection-based problem, single efficient unit, mixed binary linear programming (MBLP), relaxation problem

Received: December 29, 2020; accepted: November 2, 2021; available online: December 23, 2021

DOI: $10.17535 /$ crorr.2021.0011

\section{Introduction}

Data envelopment analysis (DEA) is a prominent non-parametric approach to evaluate the performance of a set of homogeneous decision-making units (DMUs) with multiple inputs and multiple outputs which was initially proposed by Charnes, Cooper and Rhodes [4]. In this method, the efficiency measure of each DMU is a scalar ranging between zero and one. A unit with the maximum efficiency score of 1 is efficient and otherwise, inefficient. To increase the discriminating power of the DEA approach, some ranking approaches have been developed for fully ranking efficient DMUs. However, selection-based problems lie with selecting an efficient unit among all efficient units. Toloo and Nalchigar [6] suggested the first integrated mixed binary linear programming (MBLP) model for selecting a single efficient unit with variable returns-to-scale (VRS) technology. Toloo [5] pointed out that the model of Toloo and Nalchigar [6] has fallen short in its attempt to select a single efficient DMU and then the author formulated a new MBLP model in order to overcome the shortcoming. This study demonstrates that the

${ }^{*}$ Corresponding author. 
linear programming (LP) relaxation of Toloo's model is also able to opt for a single efficient unit. More precisely, it is proven that the relaxed mixed binary linear programming (RMBLP) model is equivalent to the MBLP model of Toloo [5]. Note that a relaxed model is a regular LP model which is obtained by replacing any binary variable $\theta$ with the continuous range of $0 \leq \theta \leq 1$. In contrast with other selecting models, the proposed relaxed model can be straightforwardly solved using the simplex method which is a viable and popular tool for solving LP models. It is evident that the relaxation models are simpler, more practical, more reliable, and more succinct. It should highlighted here that the relaxation approach can extend for unit-selecting DEA models in the literature.

The return to scale describes the behavior of the rate of increase in outputs relative to the associated increase in the inputs in the long run. The first DEA model was built based on the assumption of constant returns to scale (CRS) of DMUs and then Banker, Charnes, and Cooper [3] extended a DEA model for estimating technical and scale inefficiencies under VRS assumption. In other words, the CCR model assumes that outputs increase by that same proportional change as all inputs change. Meanwhile, in the BCC model, this proportion can be increasing and decreasing as well. Note that the relaxation approach can extend to DEA selecting models in the literature. Akhlaghi and Rostamy [1] introduced an input-oriented linear model for selecting the most BCC-efficient DMU under variable returns to scale (VRS) and proposed a new LP model for finding the most BCC-efficient DMU. Using the proposed model, the decision-maker is able to find the most BCC-efficient DMU by solving only one LP.

Suppose that there are $n$ DMUs $\left(\operatorname{DMU}_{j} ; j=1, \ldots, n\right)$ to be evaluated in terms of $m$ inputs $x_{j}=\left(x_{1 j}, \ldots, x_{m j}\right)$ and $s$ outputs $y_{j}=\left(y_{1 j}, \ldots, y_{s j}\right)$. The following BCC model evaluates the performance of DMU under evaluation, i.e., $\mathrm{DMU}_{o}$ under VRS technology:

$$
\begin{array}{ll}
\max e_{o}=\sum_{r=1}^{s} u_{r} y_{r o}+u_{0}, & \\
\text { s.t. } & \\
\sum_{i=1}^{m} v_{i} x_{i o}=1, & \\
\sum_{r=1}^{s} u_{r} y_{r j}+u_{0}-\sum_{i=1}^{m} v_{i} x_{i j} \leq 0 & j=1, \ldots, n \\
v_{i} \geq \epsilon & i=1, \ldots, m \\
u_{r} \geq \epsilon & r=1, \ldots, s \\
u_{0} \text { is free. } &
\end{array}
$$

where $v_{i}$ and $u_{r}$ are the $i$ th input and $r$ th output weights, respectively; $u_{0}$ is employed to portray the situation of returns to scale, and $\epsilon$ is the non-Archimedean epsilon which prevents the weights from being zero (for more details, see Amin and Toloo[2]. DMU $\mathrm{D}_{o}$ is BCC-efficient if the optimal objective value for model (1) is 1, otherwise, it is BCC-inefficient. It should be mentioned here that usually there are many BCC-efficient DMUs that follow the lack of discriminating power of the BCC model. There is a wide range of studies that have been undertaken to address this issue [7]. Toloo [5] built the following integrated MBLP model to find a single efficient DMU under VRS assumption: 


$$
\begin{array}{ll}
\begin{array}{l}
\min d_{\text {max }}, \\
\text { s.t. }
\end{array} & \\
\sum_{i=1}^{m} v_{i} x_{i j}=1, & j=1, \ldots, n \\
\sum_{r=1}^{s} u_{r} y_{r j}-u_{0}-\sum_{i=1}^{m} v_{i} x_{i j}+d_{j}=0, & j=1, \ldots, n \\
d_{\text {max }}-d_{j} \geq 0 & \\
\sum_{j=1}^{n} \theta_{j}=n-1, & j=1, \ldots, n \\
d_{j} \leq M \theta_{j} & \\
\theta_{j} \leq N d_{j} & j=1, \ldots, n \\
d_{j} \geq 0, \theta_{j} \in\{0,1\} & j=1, \ldots \\
v_{i} \geq \epsilon & j=1, \ldots, n \\
u_{r} \geq \epsilon & i=1, \ldots, m \\
d_{\text {max }} \geq 0 & r=1, \ldots, s \\
u_{0} \text { is free. } &
\end{array}
$$

$M$ and $N$ are large positive numbers; $d_{j}$ is deviation of $\mathrm{DMU}_{j}$ from efficiency; and $\theta_{j}$ is the indicator variable (i.e., a binary variable that indicates a certain state in a model; see [8]) which is imposed on the model to fulfill the following condition:

$$
d_{j} \begin{cases}=0, & \theta_{j}=0 \\ >0, & \theta_{j}=1\end{cases}
$$

Definition [5]: decision-making unit number $k\left(\mathrm{DMU}_{k}\right)$ is the efficient unit if and only if deviation variable $d_{k}^{*}=0(k=$ the number of variant $d)$.

\section{The proposed model}

This section introduces the following LP model to determine the single efficient unit with VRS technology which is achieved by replacing binary variables $\theta_{j}$ with the continuous range $0 \leq \theta_{j} \leq 1$ for $j=1, \ldots, n$ : 


$$
\begin{aligned}
& \min d_{\max }, \\
& \text { s.t. } \\
& \sum_{i=1}^{m} v_{i} x_{i j} \leq 1 \\
& j=1, \ldots, n \\
& \sum_{r=1}^{s} u_{r} y_{r j}-u_{0}-\sum_{i=1}^{m} v_{i} x_{i j}+d_{j}=0, \quad j=1, \ldots, n \\
& d_{\text {max }}-d_{j} \geq 0 \\
& \sum_{j=1}^{n} \theta_{j}=n-1 \\
& j=1, \ldots, n \\
& d_{j} \leq M \theta_{j} \\
& \theta_{j} \leq N d_{j} \\
& \theta_{j} \leq 1 \\
& v_{i} \geq \epsilon^{*} \\
& u_{r} \geq \epsilon^{*} \\
& d_{j}, \theta_{j} \geq 0 \\
& d_{\text {max }} \geq 0 \\
& j=1, \ldots, n \\
& j=1, \ldots, n \\
& j=1, \ldots, n \\
& i=1, \ldots, m \\
& r=1, \ldots, s \\
& j=1, \ldots, n
\end{aligned}
$$

$u_{0}$ is free.

Here, $\theta_{j} \leq \min \left\{1, N d_{j}\right\}$ for $j=1, \ldots, n$ and, hence, $N$ should be large enough to allow $\theta_{j}$ for $j=1, \ldots, n(j \neq k)$ to take the value 1 ; otherwise, the model is infeasible due to the constraint $\sum_{j=1}^{n} \theta_{j}=n-1$. As a result, a feasible model can be achieved if $1=\min \left\{1, N d_{j}\right\}$ or equivalently $N \geq \max \left\{\frac{1}{d_{j}} \mid j=1, \ldots, n\right\}$ is selected. The following theorems validate the suggested relaxed model (3). The first theorem proves that the model opts for a single efficient DMU and the next theorem states that the MBLP model of Toloo [5] is equivalent to its relaxed model.

Theorem 1. Solving model (3) provides only a single efficient DMU.

Proof. Let $\left(d_{\max }^{*}, d^{*}, v^{*}, u^{*}, u_{0}^{*}, \theta^{*}\right)$ be an optimal solution to model (3). If there exists at least one zero deviation variable, e.g., $d_{k}^{*}=0$, then according to the following constraint

$$
\sum_{r=1}^{s} u_{r} y_{r k}+u_{0}-\sum_{i=1}^{m} v_{i} x_{i k}+d_{k}=0
$$

$\mathrm{DMU}_{k}$ is efficient. On the contrary, suppose that for all $j$ we have $d_{j}^{*}>0$. Let

$$
d_{\text {min }}=\min \left\{d_{j}^{*}: j=1, \ldots, n\right\} \& \bar{d}_{j}^{*}=d_{j}-d_{\text {min }}, j=1, \ldots, n
$$

It is evident that $\left(d_{\max }^{*}-d_{\min }, d^{*}, v^{*}, u^{*}, u_{0}^{*}-d_{\min }, \theta^{*}\right)$ is also feasible for model (3) with an objective value, $d_{\max }^{*}-d_{\min }$, which is less than the optimal objective value $d_{\max }^{*}$, which is impossible. As a result, model (3) is proved to provide at least one efficient unit under the VRS form of the technology. Moreover, suppose that both $\mathrm{DMU}_{k}$ and $\mathrm{DMU}_{l}$ are efficient. Hence, $d_{k}^{*}=d_{l}^{*}=0$ which, along with the constraint $\theta_{j} \leq N d_{j}$ (for $j \in\{k, l\}$ ), lead to $\theta_{k}^{*}=\theta_{l}^{*}=0$. As a result, $\sum_{j=1(j \neq k \& j \neq l)}^{n} \theta_{j} \leq n-2$ which is a contradiction to constraint $\sum_{j=1}^{n} \theta_{j}=n-1$. Therefore, it is concluded that there exists one and only one efficient DMU, which completes the proof. 
Theorem 2. The LP Model (3) is equivalent to the MBLP model (2).

Proof. Let $\left(d_{\text {max }}^{*}, d^{*}, v^{*}, u^{*}, u_{0}^{*}, \theta^{*}\right)$ be optimal solution to model (3). In the same manner analogous to the proof of Theorem 2, there exists a single zero deviation variable $d_{k}^{*}=0$ and a single zero variable $\theta_{k}^{*}=0$. Note that from the constraint $\sum_{j=1}^{n} \theta_{j}=n-1$, the only possible solution is $\theta_{j}^{*}=1, \forall j \neq k$. As a result, $\left(d_{\text {max }}^{*}, d^{*}, v^{*}, u^{*}, u_{0}^{*}, \theta^{*}\right)$ is a feasible solution for model (2). On the contrary, suppose that $\left(\bar{d}_{\text {max }}^{*}, \bar{d}^{*}, \bar{v}^{*}, \bar{u}^{*}, \bar{u}_{0}^{*}, \bar{\theta}^{*}\right)$ is an optimal solution to model (2) where $\bar{d}_{\max }^{*}<d_{\max }^{*}$. As is clear, $\left(\bar{d}_{\max }^{*}, \bar{d}^{*}, \bar{v}^{*}, \bar{u}^{*}, \bar{u}_{0}^{*}, \bar{\theta}^{*}\right)$ is a feasible solution for model (3) which is impossible because its objective function value $\bar{d}_{\text {max }}^{*}$ is smaller than the optimal objective function value $d_{\text {max }}^{*}$. In much the same way, the revise can be proved. The proof is now completed.

\section{Application}

In this paper, the feature of the model is to get the best performance without the rankings. This paper proposes a new LP model for finding the single efficient DMU. In comparison with the existing DEA, the proposed alternative LP model is more practical and more reliable and contains only essential constraints and decision variables. To find the single efficient FLD (s) given in Table1, the authors applied the proposed model [5] with $\epsilon=0.00026$. To validate the proposed model, a well-known numerical illustration is used from the DEA literature. Table1 demonstrates the real dataset of 19 facility layout designs (FLDs) with two inputs and four outputs.

\begin{tabular}{|c|cc|cccc|}
\hline FLDs & Inputs & & Outputs & & & \\
& Cost & Adjacency & Shape ratio & Flexibility & Quality & Utility \\
\hline \hline 1 & 20309.56 & 6405 & 0.4697 & 0.0113 & 0.0410 & 30.89 \\
2 & 20411.22 & 5393 & 0.4380 & 0.0337 & 0.0484 & 31.34 \\
3 & 20280.28 & 5294 & 0.4392 & 0.0308 & 0.0653 & 30.26 \\
4 & 20053.20 & 4450 & 0.3776 & 0.0245 & 0.0638 & 28.03 \\
5 & 19998.75 & 4370 & 0.3526 & 0.0856 & 0.0484 & 25.43 \\
6 & 20193.68 & 4393 & 0.3674 & 0.0717 & 0.0361 & 29.11 \\
7 & 19779.76 & 2862 & 0.2854 & 0.0245 & 0.0846 & 25.29 \\
8 & 19831.00 & 5473 & 0.4398 & 0.0113 & 0.0125 & 24.80 \\
9 & 19608.43 & 5161 & 0.2868 & 0.0674 & 0.0724 & 24.45 \\
10 & 20038.10 & 6078 & 0.6624 & 0.0856 & 0.0653 & 26.45 \\
11 & 20330.68 & 4516 & 0.3437 & 0.0856 & 0.0638 & 29.46 \\
12 & 20155.09 & 3702 & 0.3526 & 0.0856 & 0.0846 & 28.07 \\
13 & 19641.86 & 5762 & 0.2690 & 0.0337 & 0.0361 & 24.58 \\
14 & 20575.67 & 4639 & 0.3441 & 0.0856 & 0.0638 & 32.20 \\
15 & 20687.50 & 5646 & 0.4326 & 0.0337 & 0.0452 & 33.21 \\
16 & 20779.75 & 5507 & 0.3312 & 0.0856 & 0.0653 & 33.60 \\
17 & 19853.38 & 3912 & 0.2847 & 0.0245 & 0.0638 & 31.29 \\
18 & 19853.38 & 5974 & 0.4398 & 0.0337 & 0.0179 & 25.12 \\
19 & 20355.00 & 17402 & 0.4421 & 0.0856 & 0.0217 & 30.02 \\
\hline
\end{tabular}

Table 1: Inputs and outputs of 19 FLDs

GAMS software was used to solve model (3). Table2 summarizes the evaluated (BCC-) efficiencies, the optimal deviation, and binary variables. 


\begin{tabular}{|c|ccc|c|}
\hline FLDs & Efficiency & $d_{j}^{*}$ & $\theta_{j}$ & BCC-efficiency \\
\hline 1 & 0.9433 & 0.0404 & 0.4697 & 0.8433 \\
2 & 0.9791 & 0.0144 & 0.4380 & 0.8791 \\
3 & 0.9727 & 0.0187 & 0.4392 & 0.8727 \\
4 & 0.9584 & 0.0273 & 0.3776 & 0.8584 \\
5 & 0.9426 & 0.0374 & 0.3526 & 0.8426 \\
6 & 0.9852 & 0.0097 & 0.3674 & 0.9852 \\
7 & 0.9579 & 0.0256 & 0.2854 & 0.8579 \\
8 & 0.9021 & 0.0662 & 0.4398 & 0.9021 \\
9 & 0.8839 & 0.0768 & 0.2868 & 0.8839 \\
10 & 0.9999 & 0.0001 & 0.6624 & 0.999 \\
11 & 0.9769 & 0.0154 & 0.3437 & 0.9769 \\
12 & 0.9998 & 0.0001 & 0.3526 & 0.9998 \\
13 & 0.8460 & 0.1043 & 0.2690 & 0.8460 \\
14 & 1.0000 & 0.0000 & 0.3441 & 1.0000 \\
15 & 0.9821 & 0.0126 & 0.4326 & 0.9821 \\
16 & 0.9739 & 0.0183 & 0.3312 & 0.9739 \\
17 & 0.9998 & 0.0001 & 0.2847 & 0.8998 \\
18 & 0.8970 & 0.0710 & 0.4398 & 0.8970 \\
19 & 0.6774 & 0.3226 & 0.4421 & 0.6774 \\
\hline
\end{tabular}

Optimal weights

\begin{tabular}{|c|ccc|}
\hline$v_{1}^{*}$ & 0.000027 & $u_{1}^{*}$ & 0.237099 \\
$v_{2}^{*}$ & 0.000026 & $u_{2}^{*}$ & 0.262209 \\
& & $u_{3}^{*}$ & 0.000026 \\
& & $u_{4}^{*}$ & 0.000026 \\
\hline
\end{tabular}

Table 2: The optimal solution and efficiency scores

The optimal solution reported in Table 2 is equal to the one obtained by the MBLP model (2). In Toloo [5] model, it was required to first rank the units and determine the best among the candidate units with Toloo binary model. However, in the new model, which is a continuous linear model, the efficient unit is specified by solving only once and there are fewer limitations. For more details, see [5] (p. 5520).

\section{Conclusion}

In this paper, a relaxed model was suggested for identifying a single efficient unit under the VRA assumption. From a practical point of view, a real dataset was utilized to illustrate the applicability of the paper's approach. In Theorem 1, it was proved that the continuous linear model has only one efficient unit; meaning the unit with deviation variable $=0$ is efficient. In Theorem 2, it is proved that the optimal points in the new LP model are equivalent to the optimal points in the MBLP model of Toloo [5] and vice versa. The proposed relaxed model is linear and hence has many computational advantages over the non-linear model of Toloo. For instance, the proposed model is more suitable to deal with big data in DEA, which is very complex and needs a huge number of computations. Conclusively, the proposed model is more efficient. Considering the subjective opinions and intuitive senses of decision-makers is another interesting research direction (for more details see [7]. 


\section{References}

[1] Akhlaghi, R. and Rostamy-Malkhalifeh, M. (2019). A linear programming DEA model for selecting a single efficient unit. International journal of industrial engineering and operational research, 1(1), 60-66. http://bgsiran.ir/journal/ojs-3.1.1-4/index.php/IJIEOR/article/view/12/9.

[2] Amin, G. R. and Toloo, M. (2004). A polynomial-time algorithm for finding epsilon in DEA models. Computers and Operations Research, 31(5), 803-805. doi: 10.1016/S0305-0548(03)00072-8

[3] Banker, R. D., Charnes, A. and Cooper, W. W. (1984). Some models for estimating technical and scale inefficiencies in data envelopment analysis. Management Science, 30(9), 1078-1092. doi: $10.1287 /$ mnsc.30.9.1078

[4] Charnes, A., Cooper, W. W. and Rhodes, E. (1978). Measuring the efficiency of decision making units. European Journal of Operational Research, 2, 429-444. doi: 10.1016/0377-2217(79)90229-7

[5] Toloo, M. (2012). On finding the most BCC-efficient DMU: A new integrated MIP-DEA model. Applied Mathematical Modelling, 36(11), 5515-5520. doi: 10.1016/j.apm.2011.11.074

[6] Toloo, M. and Nalchigar, S. (2009). A new integrated DEA model for finding most BCC-efficient DMU. Applied Mathematical Modelling, 33(1), 597-604. doi: 10.1016/j.apm.2008.02.001

[7] Toloo, M., Nalchigar, S. and Sohrabi, B. (2018). Selecting most efficient information system projects in presence of user subjective opinions: a DEA approach. Central European Journal of Operations Research, 26(4), 1027-1051. doi: 10.1007/s10100-018-0549-4

[8] Williams, H. P. (2013). Model building in mathematical programming (Fifth Ed.). John Wiley and Sons, Ltd. https://www.wiley.com/enus/Model+Building+in+Mathematical+Programming\%2C+5th+Edition-p-9781118443330 